\title{
EchoGéo
}

$13 \mid 2010$

Afrique, 50 ans d'indépendance : État et territoires

\section{Acquisitions de la Bibliothèque de l'Institut de Géographie}

\section{Bernadette Joseph}

\section{OpenEdition}

Journals

Édition électronique

URL : https://journals.openedition.org/echogeo/11938

DOI : $10.4000 /$ echogeo. 11938

ISSN : 1963-1197

\section{Éditeur}

Pôle de recherche pour l'organisation et la diffusion de l'information géographique (CNRS UMR 8586)

Référence électronique

Bernadette Joseph, « Acquisitions de la Bibliothèque de l'Institut de Géographie », EchoGéo [En ligne], 13 | 2010, mis en ligne le 20 septembre 2010, consulté le 03 août 2021. URL : http:// journals.openedition.org/echogeo/11938; DOI : https://doi.org/10.4000/echogeo.11938

Ce document a été généré automatiquement le 3 août 2021

EchoGéo est mis à disposition selon les termes de la licence Creative Commons Attribution - Pas d'Utilisation Commerciale - Pas de Modification 4.0 International (CC BY-NC-ND) 


\title{
Acquisitions de la Bibliothèque de l'Institut de Géographie
}

\author{
Bernadette Joseph
}

\section{Afrique}

1 ADAM, L'Afrique indienne: les minorités d'origine indo-pakistanaise en Afrique orientale, Paris ; Nairobi, Karthala ; IFRA, 2009, G 19-3530

2 ALLIBERT, Plantes et sociétés, Paris, INALCO, 2009, G 19-3531

3 BAUMGARDT, ROULON-DOKO, L'expression de l'espace dans les langues africaines, Paris, Société des africanistes, 2009, G 19-3553

4 BABO, Les jeunes, la terre et les changements sociaux en pays baoulé, Côte-d'Ivoire, Dakar ; Paris, Afri ; MAPCREPOS ; Karthala 2010, G 19-3529

5 BÉNIT-GBAFFOU, FABIYI, PEYROUX, Sécurisation des quartiers et gouvernance locale : enjeux et défis pour les villes africaines (Afrique du Sud, Kenya, Mozambique, Namibie, Nigeria), Paris ; Johannesburg, Karthala ; IFAS 2010, G 19-3533

6 CAROFF, Saison cyclonique Sud-Ouest de l'océan Indien, 2000-2001, Sainte-Clotilde (Réunion), Météo France, Direction interrégionale de la Réunion, 2002, G 19-3543

7 CHOPLIN, Nouakchott : au carrefour de la Mauritanie et du monde, Paris, Karthala ; PRODIG, 2009, G 19-3528

8 DIALLO, Les Guinéens de Dakar: migration et intégration en Afrique de l'Ouest, Paris, L'Harmattan, 2009, G 19-3534

9 GBADEGESIN, BOKO, BANO-DIALLO, Ressources naturelles et environnement en Afrique, Paris, Karthala, 2010, G 19-3555

10 IGUE, KENGNE FODOUOP, ALOKO-N'GUESSAN, Maîtrise de l'espace et développement en Afrique, Paris, Karthala, 2010, G 19-3554

11 JUHÉ-BEAULATON, Forêts sacrées et sanctuaires boisés : des créations culturelles et biologiques (Burkina Faso, Togo, Bénin), Paris, Karthala, 2010, G 19-3541 
KADIEBWE, TSHIYEMBE, La guerre de six jours à Kisangani: leçons géopolitiques et géostratégiques, Paris, L'Harmattan, 2009, G 19-3532

LABRECQUE, Les différends territoriaux en Afrique : règlement juridictionnel, Paris, L'Harmattan, 2005, G 19-3552

LEVRAT, Culture commerciale et développement rural : l'exemple du coton au Nord-Cameroun depuis 1950, Paris, L'Harmattan, 2010, G 19-3535

MÉRINO, Déchets et pouvoirs dans les villes africaines : l'action publique de gestion des déchets à Nairobi de 1964 à 2002, Pessac, Maison des sciences de l'homme d'Aquitaine, 2010, G 19-3547

PASSARGE, ELDRIDGE, Adamawa : rapport de l'expédition du comité allemand pour le Cameroun au cours des années 1893-1894, Paris, Karthala, 2010, G 19-3548

RAJERIARISON, URFER, RASOANASY, Madagascar, Paris, Le Cavalier bleu, 2010, G 19-3542

RICHER, L'offensive chinoise en Afrique, Paris, Karthala, 2008, G 19-3536

SÉJOURNÉ, ANDRIAMANALINA, ANDRIANAVALONA, Madagascar, Paris, Ubifrance, 2010, G 19-3546

ZORGBIBE, Paix et guerres en Afrique. Tome 1, Un continent en dehors de l'histoire ?, Paris, Bourin, 2009, G 19-3551-<1>

\section{Amérique du nord}

CONZEN,The making of the American landscape, 2nd ed., New York, Routledge, 2010, G 20-1651

COURMONT, DAVID, États-Unis, les défis d'Obama : vers un nouveau leadership américain?, Paris, Le Félin-Kiron, 2009, G 20-1649

DAVIS, City of quartz : Los Angeles, capitale du futur, Paris, La Découverte, 2000, G 20-1657

GRANGER, Lieux d'Amérique, Lyon, Presses universitaires de Lyon, 2010, G 20-1647

Hypoxia in the northern Gulf of Mexico, New York; London, Springer, 2009, G 20-1652

Les impacts de l'exploitation du gaz et du pétrole sur les peuples autochtones de l'Arctique, Paris, L'Harmattan, 2008, G 20-1654

MAUMI, Usonia ou Le mythe de la ville-nature américaine, Paris, Éd. de la Villette, 2008, G 20-1653

QUANQUIN, LORRE JOHNSTON, FERRÉ-RODE, Comment comparer le Canada avec les EtatsUnis aujourd'hui : enjeux et pratiques, Paris, Presses Sorbonne Nouvelle, 2009, G 20-1648

SNÉGAROFF, Faut-il souhaiter le déclin de l'Amérique, Paris, Larousse, 2009, G 20-1650

VALANTIN, Hollywood, le Pentagone et Washington: les trois acteurs d'une stratégie globale, Nouvelle édition, Paris, Autrement, 2010, G 20-1659

\section{Amérique latine}

31 ALLEN,An industrial geography of cocaine, New York; London, Routledge, 2005, G 21-1718

L'Amazonie, un demi-siècle après la colonisation, Versailles, Quae, 2010, G 21-1721 
COUFFIGNAL, Amérique latine : une Amérique latine toujours plus diverse, Édition 2010, Paris, La Documentation française, 2010, G 21-1720

DABÈNE, L'Amérique latine, Paris, Le Cavalier bleu, 2009, G 21-1724

FOYER, LE BOT, Il était une fois la bio-révolution : nature et savoirs dans la modernité globale, Paris, Presses universitaires de France ; Le Monde, 2010, G 21-1723

LE DÉAUT, PROCACCIA, Rapport sur les impacts de l'utilisation de la chlordécone et des pesticides aux Antilles : bilan et perspectives d'évolution, Paris, Assemblée nationale ; Sénat, 2009, G 21-1722

POLICE, GRENAND, TOSTES, €udorado : le discours brésilien sur la Guyane française, Matoury (Guyane) : Ibis Rouge, 2010, G 21-1719

1995, l'année de tous les cyclones, Paris, Météo-France, 1995, G 21-1725

\section{Asie, Océanie}

ATTANÉ, En espérant un fils... la masculinisation de la population chinoise, Paris, Institut national d'études démographiques, 2010, G 17-2737

BAE, Regards interculturels vers l'Asie : Chine, Corée, Japon, Belfort-Montbéliard, Université de technologie de Belfort-Montbéliard, 2007, G 17-2744

BALME, La Chine, 2e édition, Paris, Le Cavalier Bleu, 2008, G 17-2764

BARZANI, Le Kurdistan d'Irak : 1918-2008, Paris, L'Harmattan, 2009, G 17-2768

BOZARSLAN, Conflit kurde : le brasier oublié du Moyen-Orient, Paris, Autrement, 2009, G 17-2766

CABESTAN, La politique internationale de la Chine : entre intégration et volonté de puissance, Paris, Presses de Sciences Po, 2010, G 17-2760

CHEVRIER, ROUX, XIAO PLANES, Citadins et citoyens dans la Chine du XXe siècle : essais d'histoire sociale, Paris, Éd. de la Maison des sciences de l'homme, 2010, G 17-2761

CONNELL,The global health care chain : from the Pacific to the world, New York; London, Routledge, 2009, G 17-2746

COURMONT, Taïwan, de l'autre côté du détroit : une île en quête d'identités, Paris, Félin, 2008, G 17-2738

The European Union and Central Asia: the new partnership in action, Luxembourg, European Communities, 2009, G 17-2733

FAUGÈRE, MERLE, La Nouvelle-Calédonie, vers un destin commun ?, Paris, Karthala, 2010, G 17-2736

FEKI, L'axe irano-syrien : géopolitique et enjeux, Paris, Studyrama, 2007, G 17-2735

FEKI, L'Iran et le Moyen-Orient : constats et enjeux, Paris, Studyrama, 2010, G 17-2734

JAVARY, WANG, La Chine nouvelle : "être riche est glorieux", Paris, Larousse, 2006, G 17-2748

53 JAUFFRET, Afghanistan 2001-2010 : chronique d'une non-victoire annoncée, Paris, Autrement, 2010, G 17-2741

HOURCADE, Géopolitique de l'Iran, Paris, A. Colin, 2010, G 17-2758

KAZANCIGIL, La Turquie, Paris, Le Cavalier Bleu, 2008, G 17-2765 


\section{Atlas}

\section{Bibliographies, documentation}



XVIII-XX siècles, Neuchâtel ; Toulouse ; Belfort, Editions Alphil-Presses universitaires suisses ; Méridiennes, Université de Toulouse II-Le Mirail FRAMESPA ; Pôle éditorial multimédia de l'UTBM, 2010, G 3-3158

MESGUICH, THOMAS, Net recherche 2010 : le guide pratique pour mieux trouver l'information utile et surveiller le web, 4e édition mise à jour, Paris, ADBS, 2010, G 1-841

Numériser et mettre en ligne, Villeurbanne, Presses de l'Enssib, 2010, G 1-837

Penser et construire l'Europe au XXe siècle, Paris, Belin, 2007, G 1-839

ROSELLI, PERRENOUD, Du lecteur à l'usager : ethnographie d'une bibliothèque universitaire, Toulouse, Presses universitaires du Mirail, 2010, G 1-844

SPITZ, HOURCADE, LALOË, Longévité de l'information numérique: les données que nous voulons garder vont-elles s'effacer?, rapport du groupe PSN (Pérennité des Supports Numériques) commun à l'Académie des sciences et à l'Académie des technologies, Les Ulis, EDP Sciences, 2010, G 1-835

\section{Colloques}

L'action publique face à la mobilité, Paris, L'Harmattan, 2010, G 3-3160

AKINYEYE, BARRY, SANÉ,Nation-states and the challenges of regional integration in West Africa, Paris, Karthala, 2010, G 3-3150

BARONE-VISIGALLI, ROOSEVELT, POLICE, Amaz'hommes : sciences de l'homme et sciences de la nature en Amazonie : colloque organisé par le PUG, pôle universitaire de Guyane et les chercheurs du CRILLASH, institut d'enseignement supérieur de la Guyane, université des Antilles et de la Guyane, à Cayenne, les 17, 18 et 19 avril 2009, Matoury, Ibis rouge, 2010, G 3-3159

BAUBÉROT, BOURILLON, Urbaphobie ou la détestation de la ville aux XIXe et XXe siècles : actes du colloque réuni à Paris-12 Val-de-Marne, les 8 et 9 mars 2007, Pompignac, Bière, 2009, G 3-3183

BELOT, BIZERN, Frontières en images : une mémoire cinématographique, colloque, Belfort, 1-2 décembre 2005, Belfort, Université de technologie Belfort-Montbéliard, 2006, G 3-3157

BOUQUET, Les géographes et le développement : discours et actions, Pessac, Maison des sciences de l'homme d'Aquitaine, 2010, G 3-3166

BOURGUINAT, Le voyage au féminin: perspectives historiques et littéraires: XVIIIe-XXe siècles, Strasbourg, Presses Universitaires de Strasbourg, 2008, G 3-3151

CAMIADE, ALMARCHA, L'Albera : terre de passage, de mémoires et d'identités = terra de pas, de memòries $i$ d'identitats : actes du colloque de Banyuls-sur-Mer, 3-4 mai 2005, Perpignan, IFCTPUP, 2006, G 3-3177

CHAUVARD, LEBEAU, Éloignement géographique et cohésion familiale: XVe-XXe siècle, Strasbourg, Presses universitaires de Strasbourg, 2006, G 3-3152

DAUMAS, LAMARD, TISSOT, Histoires de territoires : les territoires industriels en question

DUMOND, DURAND, L'olivier dans l'Europe méditerranéenne de l'Antiquité à nos jours : terroir, paysage et économie : actes du colloque tenu à Montpellier les 17 et 18 mars 2006, Montpellier, Presses universitaires de la Méditerranée, 2007, G 3-3182 
3-3174

Les échelles: pour les géographes et les autres: cultures, finalités et pratiques scalaires : livre des Les espaces collectifs dans les campagnes XIe-XXIe siècle : actes du colloque, Université BlaisePascal de Clermont-Ferrand, 15-17 mars 2004, Clermond-Ferrand, Presses universitaires Blaise-Pascal, 2007, G 3-3179

FRAY, PÉROL, L'historien en quête d'espaces : actes du colloque, Clermont-Ferrand, printemps 2002, Clermont-Ferrand, Presses universitaires Blaise-Pascal, 2004, G 3-3178

GÉLARD, CHEMIN, L'émergence de la puissance indienne : mythes et réalités, Rennes, Presses universitaires de Rennes, 2010, G 3-3180

HERVIEU, HUBERT, Sciences en campagne : regards croisées, passés et à venir, La Tourd'Aigues, Éd. de l'Aube, 2009, G 3-3176

KHOURY, Palestine-Israël : 60 ans de conflit, Pessac, Presses universitaires de Bordeaux, 2010, G 3-3153

MARTENS, L'Allemagne, vingt ans après la fin de la guerre froide et son unification, Pessac, Presses universitaires de Bordeaux, 2010, G 3-3169

ORTOLANI, VERNIER, BOTTIN, Pouvoirs et territoires dans les États de Savoie: actes du colloque international de Nice, 29 novembre-1er décembre 2007, Nice, Serre, 2010, G 3-3149

PAULIAT, L'autonomie des collectivités territoriales en Europe: une source potentielle de conflits ?, Limoges, Pulim, 2004, G 3-3155

Quel avenir pour l'intégration européenne? Regards croisés franco-allemands sur le traité de Lisbonne, Tours, Presses universitaires François-Rabelais, 2010, G 3-3154

RAYNAL, Roms, tsiganes et gens du voyage, Montrouge ; Paris, Centre de ressources VilleEcole-Intégration ; Scérén-CNDP, 2009, G 3-3148

SIROST, La vie au grand air : aventures du corps et évasions vers la nature, Nancy, Presses universitaires de Nancy, 2009, G 3-3147

TÜRKMEN, Turquie, Europe : le retour des nationalismes ?, Paris ; Istanbul, L'Harmattan ; Université Galatasaray, 2010, G 3-3170

VIAUT, LAMUELA, Variable territoriale et promotion des langues minoritaires, Pessac, Maison des sciences de l'Homme d'Aquitaine, 2007, G 3-3161

WESTPHAL, FLABBI, Espaces, tourismes, esthétiques, Limoges, Pulim, 2010, G 3-3181

\section{Dictionnaire}

BELZACQ, MIOTTO, PUTFIN, Dico atlas des États du monde, Nouvelle édition actualisée, Paris, Belin, 2009, W 406

RAPOPORT, Penser et construire l'Europe : outils, Neuilly-sur-Seine, Atlande, 2007, W 405

TANGUY, DECOBECQ, Dictionnaire des volcans, Paris, J.-P. Gisserot, 2009, W 407

\section{Europe}

Agricultural statistics : main results, 2007-08, 2009 edition, Luxembourg, Office for official publications of the European communities, 2009, G 15-6105 
11 AMAR, MICHAUD, La marche au cœur des mobilités : état des connaissances, Lyon, CERTU, 2009, G 15-6091

ANGRAND, MARX, L'Allemagne, 2e édition, Paris, Le Cavalier bleu, 2009, G 15-6125

AVGERI, MAGNILLAT, Enjeux et rouages de l'Europe actuelle: culture et citoyenneté européennes, [7e édition], Vanves, Foucher, 2009, G 15-6084

BAÏSSAS, IACOMMELI, ARAMBURU, Royaume-Uni, Édition 2010-2011, Paris, Ubifrance, 2009, G 15-6065

BARBE, GISCARD D'ESTAING, L'espace judiciaire européen, Paris, La Documentation française, 2007, G 15-6087

BERTRAND, Le conflit helléno-turc : la confrontation des deux nationalismes à l'aube du XXIe siècle, Paris, Maisonneuve \& Larose, 2004, G 15-6127

BRIENS, MOHNIKE, Capitales culturelles et Europe du Nord, Strasbourg, Université de Strasbourg, Départements d'études néerlandaises et scandinaves, 2009, G 15-6136

CAMP-PIETRAIN, La devolution : Écosse - Pays de Galles, Neuilly-sur-Seine, Atlande, 2006,

G 15-6088

CARRÈRE D'ENCAUSSE, La Russie entre deux mondes, Paris, Fayard, 2010, G 15-6138

CHALTIEL-TERRAL, SAURON, Le processus européen de décision après le traité de Lisbonne, 2e édition, Paris, La Documentation française, 2010, G 15-6123

Combating poverty and social exclusion : a statistical portrait of the European Union 2010, 2010 ed., Luxembourg, Office for official publications of the European Communities, 2010, G 15-6108

CRESPY, PETITHOMME, L'Europe sous tensions : appropriation et contestation de l'intégration européenne, Paris, L'Harmattan, 2009, G 15-6067

Demography report 2008 : meeting social needs in an ageing society : Commission staff working document, Luxembourg, Office for official publications of the European Communities, 2009, G 15-6114

DELORCA, Abkhazie : à la découverte d'une "République" de survivants, Paris, Éd. du Cygne, 2010, G 15-6139

DRÉANO, VACARISAS, Guerres et paix au Caucase : empires, peuples et nations, Paris, Non Lieu, 2009, G 15-6129

DUBOS, KAUFFMANN, L'Europe des services : l'approfondissement du marché intérieur, Paris, Pédone, 2009, G 15-6086

DUROUX, Les Auvergnats de Castille : renaissance et mort d'une migration au XIXe siècle, Clermont-Ferrand, Association des publications de la Faculté des Lettres et Sciences humaines de Clermont-Ferrand, 1992, G 15-6140

ELLERMAN, CONVERY, PERTHUIS, Le prix du carbone : les enseignements du marché européen du CO2, Paris, Pearson Education France, 2010, G 15-6095

L'éducation et l'accueil des jeunes enfants en Europe: réduire les inégalités sociales et culturelles, Bruxelles, Eurydice, 2009, G 15-6061

EMELIANOFF, STEGASSY, Les pionniers de la ville durable : récits d'acteurs, portraits de villes en Europe, Paris, Autrement, 2010, G 15-6070 

15-6117 15-6137 2009, G 15-6102

L'enseignement supérieur en Europe 2009, les avancées du processus de Bologne, Bruxelles, Eurydice, 2009, G 15-6060

L'état de l'Union 2010 : rapport Schuman sur l'Europe, Paris, Lignes de repères, 2010, G

Europe's demographic future : facts and figures on challenges and opportunities, Luxembourg, Office for Official Publications of the European Communities, 2007, G 15-6113

Les évaluations standardisées des élèves en Europe: objectifs, organisation et utilisation des résulats, Bruxelles, Agence exécutive éducation, audiovisuel et culture, 2009, G 15-6059

FAVAREL-GARRIGUES, ROUSSELET, La Russie contemporaine, Paris, Fayard, 2010, G

Forestry statistics, 2007 édition, Luxembourg, Office for official publications of the European Communities, 2007, G 15-6106

La Forêt de Soignes : connaissances nouvelles pour un patrimoine d'avenir, Wavre, Mardaga,

FORET, ITÇAINA, Dieu loin de Bruxelles: l'européanisation informelle du religieux, Paris, L'Harmattan, 2008, G 15-6072

Greenhouse gas emission trends and projections in Europe 2007 : tracking progress towards Kyoto targets, Copenhage ; Luxembourg, EEA ; Office for Official Publications of the European Communities, 2007, G 15-6110

GRUEL-DIEUDÉ, Chypre et l'Union européenne : mutations diplomatiques et politiques, Paris, L'Harmattan, 2007, G 15-6128

GUILLAUME, Penser et construire l'Europe de 1919 à 1992 : manuel et dissertations corrigées, Paris, Ellipses, 2007, G 15-6075

HAËNTJENS, Urbatopies : ces villes qui inventent l'urbanisme du XXIe siècle, La Tourd'Aigues, Éd. de l'Aube, 2010, G 15-6096

HAUGHTON,The new spatial planning : territorial management with soft spaces and fuzzy boundaries, London ; New York, Routledge, 2010, G 15-6092

HILLARD, La décomposition des nations européennes : de l'union euro-atlantique à l'État mondial, $2 e$ édition augmentée, Paris, F.-X. de Guibert, 2010, G 15-6135

HINNEWINKEL, Les terroirs viticoles : origines et devenirs, Paris, Féret, 2004, G 15-6098

KAHN, Géopolitique de l'Union européenne, Paris, A. Colin, 2007, G 15-6080

LABRECQUE, Les différends territoriaux en Europe : jurisprudence de la Cour internationale de justice, Paris, L'Harmattan, 2009, G 15-6130

LACONTE, COULIE, La recherche de la qualité environnementale et urbaine : le cas de Louvainla-Neuve (Belgique), Lyon, CERTU, 2009, G 15-6076

LÉVY-VROELANT, TUTIN, Le logement social en Europe au début du XXIe siècle : la révision générale, Rennes, Presses universitaires de Rennes, 2010, G 15-6141

LIÉGEOIS, Roms en Europe, Strasbourg, Éd. du Conseil de l'Europe, 2007, G 15-6066

Lignes directrices: droits de l'homme et droit international humanitaire, Luxembourg, office des publications officielles des Communautés européennes, 2009, G 15-6063

MAJERUS, Dépasser le cadre national des "Lieux de mémoire" : innovations méthodologiques, approches comparatives, lectures transnationales $=$ methodologische Innovationen, 
vergleichende Annäherungen, transnationale Lektüren, Bruxelles ; Berlin, PIE P. Lang, 2009, G 15-6134

MALTERRE, PRADEAU, L'Europe en fiches, 6e édition, Rosny, Bréal, 2008, G 15-6071

Manuel européen sur les données relatives à l'égalité: pourquoi et comment élaborer un socle national de connaissances sur l'égalité et la discrimination fondée sur la race ou l'origine ethnique, la religion ou les convictions, un handicap, l'âge ou l'orientation sexuelle, Luxembourg, Office des publications officielles des Communautés européennes, 2007, G 15-6055

MARCHAND, SUSS, HERBAUT, Atlas Moscou, Paris, Autrement, 2010, G 15-6054

Marchesin, La Biélorussie, Paris, Karthala, 2006, G 15-6094

MASBOUNGI, MANGIN, GRAVELAINE, ROUX, FORTIER, Agir sur les grands territoires, Paris, Le Moniteur, 2009, G 15-6077

MAURER, Les espaces de l'Allemagne au XIXe siècle: frontières, centres et question nationale, Strasbourg, Presses universitaires de Strasbourg, 2010, G 15-6085

Meilleures pratiques Leader+, Luxembourg, Office des publications officielles des communautés européennes, 2008, G 15-6111

MONCEAU, L'Europe au miroir de la Turquie, Paris, L'Harmattan, 2009, G 15-6073

MONGRENIER, LACOSTE, La Russie menace-t-elle l'Occident?, Paris, Choiseul, 2009, G 15-6064

MORICE, POTOT, JOUNIN, De l'ouvrier immigré au travailleur sans papiers : les étrangers dans la modernisation du salariat, Paris, Karthala, 2010, G 15-6068

Moving Europe : EU research on migration and policy needs, Luxembourg, Office for official publications of the european communities, 2009, G 15-6112

NOËL, JEANNESSON, BRICE, ULRICH-PIER, Penser et construire l'Europe (1919-1992), Neuilly, Atlande, 2008, G 15-6081

OGILVIE, SARDINHA, Wolf, Vivre en Europe : philosophie, politique et science aujourd'hui, Paris, L'Harmattan, 2010, G 15-6115

PFEIL, Football \& identité en France et en Allemagne, Villeneuve d'Ascq, Presses universitaires du Septentrion, 2010, G 15-6090

POIRRIER, MONNIER, Paysages des campus : urbanisme, architecture et patrimoine, Dijon, Éd. universitaires de Dijon, 2009, G 15-6089

Protection des eaux souterraines en Europe : la nouvelle directive sur les eaux souterraines, une consolidation du cadre réglementaire de l'UE, Luxembourg, Office des publications officielles des Communautés européennes, 2008, G 15-6062

RAYNER, L'Italie, Paris, Le Cavalier bleu, 2009, G 15-6124

Regions of the European Union : a statistical portrait, 2009 edition, Luxembourg, Office for official publications of the European communities, 2008, G 15-6107

ROBERT, Mesurer la délinquance en Europe : comparer statistiques officielles et enquêtes, Paris, L'Harmattan, 2009, G 15-6132

Roumanie, Éd. 2010-2011, Paris, Ubifrance, 2010, G 15-6116

SAINT POL, Le corps désirable: hommes et femmes face à leur poids, Paris, Presses universitaires de France, 2010, G 15-6069 
SNÉGAROFF, L'Europe, Paris, Ellipses, 2008, G 15-6082

Stratégie de l'AEE 2009-2013: programme de travail pluriannuel, Copenhague, AEE, 2009, G 15-6058

STEFFEN, Les États face au sida en Europe, Grenoble, Presses universitaires de Grenoble, 2001, G 15-6101

SUR, CHATRÉ, DELORY, Conflits et sécurité dans l'espace Mer Noire: l'Union européenne, les riverains et les autres, Paris, Éd. Panthéon-Assas, 2010, G 15-6078

THERBORN, ZAGRODZKI, Les sociétés d'Europe du XXe au XXIe siècle : la fin de la modernité européenne ?, Paris, A. Colin, 2009, G 15-6126

TERPAN, La politique étrangère, de sécurité et de défense de l'Union européenne, Paris, La Documentation française, 2010, G 15-6074

TEURTRIE, Géopolitique de la Russie : intégration régionale, enjeux énergétiques, influence culturelle, Paris, L'Harmattan, 2010, G 15-6133

Using statistics to calculate greenhouse gas emissions : a statical guide, 2010 ed., Luxembourg, Office for Official Publications of the European Communities, 2010, G 15-6109

VERBEKEN, DE WINNE, VIGNAUX, VAN ISTENDAEL, La terre promise (Flamands en Wallonie) : essai, Bègles, le Castor astral, 2010, G 15-6079

VEYRIER, L'Union européenne et ses relations de voisinage, Paris, Direction des Journaux officiels, 2009, G 15-6093

ZAUBERMAN, Victimation et insécurité en Europe : un bilan des enquêtes et de leurs usages, Paris, L'Harmattan, 2008, G 15-6131

\section{France}

ALBERTELLI, LEVASSEUR, CRÉMIEUX-BRILHAC, Atlas de la France libre: de Gaulle et la France libre, une aventure politique, Paris, Autrement, 2010, G 10-5899

ANDRÉ, L'offre de santé dans les collectivités ultramarines, Paris, Direction des Journaux officiels, 2009, G 10-5942

BENATSOU, Les entreprises dans les zones franches urbaines : bilan et perspectives, Paris, Direction des Journaux officiels, 2009, G 10-5940

BESSE, MAILLARD, Les immigrations en Picardie : XIXe-XXe siècles, Amiens ; Paris, Licorne ; diff. L'Harmattan 2009, G 10-5898

BIER, CHAMBON, QUEIROZ, Mutations territoriales et éducation : de la forme scolaire vers la forme éducative?, Issy-les-Moulineaux, ESF éditeur, 2010, G 10-5922

BLANC, Le Grand Paris du XXIe siècle, Paris, Le Cherche midi, 2010, G 10-5939

BLONDEL, SULLY JAULMES, Un siècle passe, 3e édition, Paris, D. Carré, 2007, G 10-5951

BOISSON, La mesure du déclassement, Paris, La Documentation française, 2009, G 10-5978

BONIFACE, La politique étrangère de Nicolas Sarkozy : rupture ou continuité ?, Paris, IRIS ; A. Colin, 2010, G 10-5954

BOUFFETTE, BONNET, Itinéraires géologiques à Rennes, Rennes, Apogée, 2009, G 10-5930

BOUILLON, AGIER, Les mondes du squat : anthropologie d'un habitat précaire, Paris, Presses universitaires de France, 2009, G 10-5955 
BOULA DE MAREÜIL, D'où viennent les accents régionaux ?, Paris, Le Pommier, 2010, G 10-5963

BOUTELET-BLOCAILLE, LARCENEUX, BARCZAK, Gouvernance de l'eau : intercommunalités et recomposition des territoires, Dijon, Éd. universitaires de Dijon, 2010, G 10-5905

BOUTRAND, Seniors et cité, Paris, Direction des Journaux officiels, 2009, G 10-5945

BRAUN, COLLIGNON, La France en fiches, 6e édition, Rosny, Bréal, 2008, G 10-5919

BRAVO ET AL., La société et l'économie à l'aune de la révolution numérique : enjeux et perspectives des prochaines décennies (2015-2025), Paris, La Documentation française, 2009, G 10-5979

CAPSIE, L'ouverture à l'urbanisation : renouvellement d'une notion fondamentale du droit de l'urbanisme, Perpignan, Presses universitaires de Perpignan, 2001, G 10-5983

CHEVASSUS-AU-LOUIS, SALLES, PUJOL, Approche économique de la biodiversité et des services liés aux écosystèmes : contribution à la décision publique, Paris, La Documentation française, 2009, G 10-5977

COUROUBLE, ARTHUS-BERTRAND, Développement durable et agroalimentaire: partage d'expériences en Basse-Normandie, Paris, Publibook, 2010, G 10-5903

DEBOUDT, LARRUE, BERSANI, Inégalités écologiques, territoires littoraux \& développement durable, Villeneuve-d'Ascq, Presses universitaires du Septentrion, 2010, G 10-5910

DION, Histoire de la vigne et du vin en France: des origines au XIXe siècle, Paris, CNRS, 2010, G 10-5901

DUPONT, LE POTTIER, IZARD, L'invention de la Haute-Garonne : géographie administrative de la Haute-Garonne de la Révolution à nos jours, Toulouse, Les Amis des Archives de la HauteGaronne, 2009, G 10-5929

DUTHILLEUL, Suivi de la situation de la France au regard des indicateurs de Lisbonne, Édition 2009, Paris, Direction des Journaux officiels, 2009, G 10-5946

EVETTE, MÉRIAUX, LAIGLE, Risques et impacts des retenues d'altitude, Versailles, Quae, 2009, G 10-5924

FAYT, Les dimensions "villageoises" à Paris, Paris, L'Harmattan, 2009, G 10-5914

Les fonds structurels européens 2007-2013, 2e édition actualisée, Paris, La Documentation française, 2010, G 10-5986

GALLON, FLATRÈS, La fracture agricole: les lobbies face à l'urgence écologique, Paris, Delachaux et Niestlé, 2008, G 10-5928

GARNIER, MORICEAU, Terre de conquêtes : la forêt vosgienne sous l'Ancien régime, Paris, Fayard, 2004, G 10-5900

GAUCHON, HUISSOUD, Vive la France quand même! Les atouts de la France dans la mondialisation : rapport Anteios 2011, Paris, Presses Universitaires de France, 2010, G 10-5970

GENDRON, Animaux et noms de lieux, Paris, Errance, 2010, G 10-5904

La gestion du trait de côte, Versailles, Quae, 2010, G 10-5912

GIRARDON, Politiques d'aménagement du territoire, 2e édition mise à jour et enrichie, Paris, Ellipses, 2010, G 10-5952 
218 G 10-5933 G 10-5897 2009, AQ 13-133 G 10-5937 10-5936

GUTTON, Établir l'identité : l'identification des français du Moyen âge à nous jours, Lyon, Presses universitaires de Lyon, 2010, G 10-5971

GWIAZDZINSKI, RABIN, Urbi et orbi : Paris appartient à la ville et au monde, Paris, Éd. de l'Aube, 2010, G 10-5972

HERZOG-EVANS, La prison dans la ville, Toulouse, Erès, 2009, G 10-5980

Innover en Ile-de-France : 32 priorités pour les PME, Paris, La Documentation française, 2007,

JACQUET, WOLIKOW, Un siècle de construction du vignoble bourguignon : les organisations vitivinicoles de 1884 aux AOC, Dijon, Éd. universitaires de Dijon, 2009, G 10-5943

KRISTEVA, Le message culturel de la France et la vocation interculturelle de la francophonie, Paris, Direction des Journaux officiels, 2009, G 10-5948

LABRUNE, La géographie de la France, Paris, Nathan, 2010, A 10.0260

LALLEMENT, WINKIN, La ville marchande, enquête à Barbès, Paris, Téraèdre, 2010,

LAMBERT, Les modalités de formation des prix alimentaires : du producteur au consommateur, Paris, Direction des Journaux officiels, 2009, G 10-5947

LENOIR, TIBERGHIEN, Territoires occupés: une autre vision de la France, Le Rheu, LME,

LESUR, Les hôtels de Paris : de l'auberge au palace, XIXe-XXe siècles, Neuchâtel, Alphil, 2005,

Le littoral en projets, Marseille, Parenthèses, 2009, G 10-5907

LEVAN-LEMESLE, Industrialisation et sociétés, 1880-1970 : la France, Paris, Ellipses, 1998, G

MALEVAL, Le lac de Saint-Pardoux : évolution du littoral et sédimentation lacustre, ClermontFerrand, Presses universitaires Blaise Pascal, 2010, G 10-5908

MALHER, LESAFFRE, Oiseaux nicheurs de Paris : un atlas urbain, Paris, CORIF ; Delachaux et Niestlé, 2010, G 10-5931

MARCON, Conditions pour le développement numérique des territoires, Paris, Direction des Journaux officiels, 2009, G 10-5944

MAUPERTUIS, La Corse et le développement durable, Ajaccio, Albiana, 2010, G 10-5960

MONGRENIER, LELLOUCHE, La France, l'Europe, l'OTAN: une approche géopolitique de l'atlantisme français, Paris, UNICOMM, 2006, G 10-5926

MONTARSOLO, L'Eurafrique, contrepoint de l'idée d'Europe : le cas français de la fin de la Deuxième Guerre mondiale aux négociations des Traités de Rome, Aix-en-Provence, Publications de l'Université de Provence, 2010, G 10-5959

NÉGRIER, DJAKOUANE, JOURDA, Les publics des festivals, Paris ; Montpellier, M. de Maule ; France festivals ; Réseau en scène Languedoc-Roussillon, 2010, G 10-5958

PASSARRIUS, CATAFAU, MARTZLUFF, Archéologie d'une montagne brûlée : massif de Rodès, Pyrénées-Orientales, Canet, Trabucaire, 2009, AQ 13-132

PAUL-DUBOIS-TAINE, Péage urbain : principes pour une loi, Paris, La Documentation française, 2009, G 10-5976 

2009, G 10-5906 10-5938 G 10-5909 2010, G 10-5981 G 10-5902 10-5941 2010, K 1083

PEUGEOT, Migrants: quelle place pour la diversité nantaise?, Nantes, Institut Kervégan,

PEYRAS, MÉRIAUX, Retenues d'altitude: évaluation des risques et des impacts, conception, réalisation, surveillance, réhabilitation, Versailles, Quae, 2009, G 10-5932

PEYROUTET, Le tourisme en France, Paris, Nathan, 2010, G 10-5925

PINON, LEMAÎTRE, Patrimoine fluvial : canaux et rivières navigables, Paris, Scala, 2009, G

POIRIER, Un espace rural à la loupe: paysage, peuplement et territoires en Berry de la préhistoire à nos jours, Tours, Presses universitaires François-Rabelais, 2010, 2009,

Quelle(s) métropole(s) pour le Grand-Ouest? Nouvelles donnes pour Nantes, Nantes, Institut Kervégan, 2009, G 10-5920

QUINET, BAUMSTARK, CÉLESTIN-URBAIN, POULIQUEN, AUVERLOT, RAYNARD, La valeur tutélaire du carbone, Paris, La Documentation française, 2009, G 10-5975

La rénovation urbaine à l'épreuve des faits : rapport 2009 du Comité d'évaluation et de suivi de l'ANRU, Paris, La Documentation française, 2010, G 10-5918

Saint-Étienne, Mobiliser les territoires pour une croissance harmonieuse : rapport au Président de la République, Paris, La Documentation française, 2009, G 10-5923

SALVAYRE, Le livre des eaux souterraines des Pyrénées catalanes : essai, Canet, Trabucaire,

SCHNEIDER, Le développement durable territorial, La Plaine-Saint-Denis, AFNOR, 2009,

SMITS, Géographie de la France, Paris, Hatier, 2007, G 10-5915

La vieillesse, Paris, Ellipses, 2010, G 10-5927

TARDY, Infrastructures et développement durable des territoires : un autre regard, une nouvelle vision, Paris, Direction des Journaux officiels, 2009, G 10-5950

VIEILLE MARCHISET, Des loisirs et des banlieues : enquête sur l'occupation du temps libre dans les quartiers populaires, Paris, l'Harmattan, 2009, G 10-5982

VIGUERIE, Les usages domestiques de l'eau, Paris, Direction des Journaux officiels, 2009, G

WALTER, Réalité et avenir du dialogue social territorial, Paris, Direction des Journaux officiels, 2009, G 10-5949

WIEL, Étalement urbain et mobilité, Paris, La Documentation française, 2010, G 10-5984

\section{Généralités (histoire, épistémologie, méthode, cartographie, SIG)}

BARD, CHAPPELLAZ, Sur les origines de l'effet de serre et du changement climatique, Montreuil, La Ville brûle, 2010, G 5-1823

BARREAU, L'épistémologie, 7e édition mise à jour, Paris, Presses universitaires de France, 

2010, G 5-1817 2010, G 5-1835 5-1852 2008, G 5-1833 5-1861 G 5-1859

BATTISTONI-LEMIÈRE, NONJON, LE FUR, BRUN, DUBUT, Cartes en mains : méthodologie de la cartographie, Paris, Ellipses, 2010, G 5-1819

BEAUD, WEBER, Guide de l'enquête de terrain: produire et analyser des données ethnographiques, 4e édition augmentée, Paris, La Découverte, 2010, G 5-1829

BONNET, GUICHARD, ANTOINE, BURGER, Histoire et géographie: composante majeure, composante mineure, Paris, Hachette éducation, 2010, G 5-1816

BOONAERT, BinG Maps: guide complet de la cartographie interactive, Saint-Herblain, ENI,

BOUDIA, RASMUSSEN, SOUBIRAN, HARTOG, DAVALLON, Patrimoine et communautés savantes, Rennes, Presses universitaires de Rennes, 2009, G 5-1826

CASTREE, ROGERS, SHERMAN, QuestioninG geography: fundamental debates, Malden Mass.; Oxford; Victoria, Blackwell, 2005, G 5-1830

CHARNAY, MANTE, DORANGE, Histoire-Géographie, instruction civique et morale (et histoire des arts) : épreuve écrite d'admissibilité, Paris, Hatier, 2010, G 5-1863

CORREIA, Mon GPS en action : créer et enrichir ses cartes avec Google Earth, Google Maps, Openstreetmap... Paris, Eyrolles, 2010, G 5-1851

CRAMPTON,Mapping: a critical introduction to cartography and GIS, Chichester (West Sussex); Malden (Mass.), Wiley-Blackwell, 2010, G 5-1834

DAVIDENKOFF, KAHN, Les universités sont-elles solubles dans la mondialisation?, Paris, Hachette littératures, 2006, G 5-1827

GERSMEHL, SOLEM,Teaching geography, Second edition, New York ; London, Guilford Press, 2008, G 5-1839, CD 350

GHILANI,Adjustment computations: spatial data analysis, 5th ed., Hoboken, N.J., J. Wiley,

GOMEZ, JONES, Research methods in geography: a critical introduction, Chichester, Malden, MA, Wiley-Blackwell, 2010, G 5-1836

JANSON, MALCZYK, 280 questions d'histoire et de géographie : concours de professeur d'école: nouveau concours : avec 101 confusions à éviter : conforme aux programmes du concours 2010, 5e édition, Tonnerre, Tempes, 2009, G 5-1828

LARRÈRE, LARRÈRE, Du bon usage de la nature : pour une philosophie de l'environnement, Paris, Flammarion, 2009, G 5-1843

LEROY, Éros géographe, Villeneuve-d'Ascq, Presses universitaires du Septentrion, 2010, G

6 LEUANGTHONG, KHAN, DEUTSCH,Solved problems in geostatistics, Hoboken, N.J., Wiley

LOISON, Français, Histoire-géographie, Instruction civique et morale : entraînement, épreuve écrite CRPE, Paris, Vuibert, 2010, G 5-1862

LOISON, Professeur des écoles : tout le cours des épreuves écrites CRPE, Paris, Vuibert, 2010, G

MANCEAU, COURTIN, Fonction publique : le guide 2010 des concours, Paris, L'Étudiant, 2010, 
MANIGLIER, La perspective du diable : figurations de l'espace et philosophie de la Renaissance à "Rosemary's baby" : sur une oeuvre de Laetitia Delafontaine et Grégory Niel, Arles ; Nice, Actes Sud ; Villa Arson, 2010, G 5-1850

MILOT, LA BRANCHE, Enseigner les sciences sociales de l'environnement: un manuel multidisciplinaire, Villeneuve d'Ascq, Presses Universitaires du Septentrion, 2010, G 5-1825

MUET, Les géographes et l'Europe : l'idée européenne dans la pensée géopolitique française de 1919 à 1939, Genève, Institut européen de l'Université de Genève, 1996, G 5-1849

NABHAN, CARO, Aux sources de notre nourriture, Nikolaï Vavilov et la découverte de la biodiversité, Bruxelles, Nevicata, 2010, G 5-1820

OBERMEYER, PINTO, Managing geographic information systems, 2nd ed., New York, Guilford Press, 2008, G 5-1838

O'SULLIVAN, UNWIN,Geographic information analysis, Second edition, Hoboken (N.J.), J. Wiley, 2010, G 5-1831

PAQUOT, Les faiseurs de villes : 1850-1950, Gollion (Suisse), Infolio, 2010, G 5-1824

PELLETIER, Le théâtre du monde : atlas d'hier, Paris, Paris bibliothèques, 2009, G 5-1818

PERRIN-VAN HILLE, L'entretien avec le jury, Nouvelle édition, Paris, La Documentation française, 2010, G 5-1754 BIS

POCHET, La rédaction d'un article scientifique : petit guide pratique adapté aux sciences appliquées et sciences de la vie à l'heure du libre accès, Gembloux (Belgique), Les Presses agronomiques de Gembloux, 2009, G 5-1865

PONCELET, L'invention des sciences coloniales belges, Paris, Karthala, 2008, G 5-1840

PRUM, La démarche statistique, Toulouse, Cépaduès, 2010, G 5-1866

RIPLEY,Spatial statistics, Hoboken N.J., J. Wiley, 1981, G 5-1832

RIST, Le développement : histoire d'une croyance occidentale, 3e édition revue et augmentée, Paris, Sciences Po, les presses, 2007, G 5-1846

RUETE, Géopositionnement et mobilités : GPS, Egnos et Galileo... Belfort, Université de Technologie de Belfort-Montbéliard, 2009, G 5-1837

TISSERAND, Épreuve écrite de français, histoire-géographie et instruction civique et morale : nouveau concours 2010 : professeur des écoles, Vanves, Foucher, 2010, G 5-1864

TRÉGUER, Trois marins pour un pôle, Versailles, Quae, 2010, G 5-1868

VINCENT, Élisée Reclus : géographe, anarchiste, écologiste, Paris, R. Laffont, 2010, G 5-1821

ZIEGLER, La représentation du paysage au cinéma, Paris, Bazaar \& Co, 2010, G 5-1867

\section{Géographie humaine}

AGIER, Gérer les indésirables: des camps de réfugiés au gouvernement humanitaire, Paris, Flammarion, 2008, G 9-7328

ANDERSON, Understanding cultural geography : places and traces, London ; New-York, Routledge, 2009, G 9-7365

L'asile au sud, Paris, La Dispute, 2008, G 9-7418 
Atlas Mediterra: agriculture, alimentation, pêche \& mondes ruraux en Méditerranée, Paris, Presses de Sciences Po, 2010, G 9-7316

Atlas Mediterra: Mediterranean agriculture, food, fisheries \& the rural world, Paris, Presses de Sciences Po, 2010, G 9-7316 BIS

L'avenir des migrations internationales vers les pays de l'OCDE, Paris, OCDE, 2009, G 9-7449

BALLET, DUBOIS, MAHIEU, L'autre développement : le développement socialement soutenable, Paris, L'Harmattan, 2005, G 9-7390

La bataille de l'énergie, Paris, La Documentation française, 2007, G 9-7402

BASTIDON GILLES, BRASSEUL, GILLES, BOISSIEU, Histoire de la globalisation financière : essor, crises et perspectives des marchés financiers internationaux, Paris, A. Colin, 2010, G 9-7294

BELZACQ, MIOTTO, PUTFIN, Dico atlas des continents, Paris, Belin, 2009, A 9.0166

BERTRAND, AMMIRATI, RENAUDEAU, EMMANUELLI, NEMITZ, Risques chimiques, accidents, attentats, Paris, Elsevier, 2006, G 9-7309

BETBÈZE, Les 100 mots de l'économie, 4e édition entièrement revue et mise à jour, Paris, Presses universitaires de France, 2010, K 1084

BLEYS, Voyage en francophonie: une langue autour du monde, Paris, Autrement, 2010, G 9-7331

BONIFACE, Dictionnaire incorrect de l'état du monde, Paris, Larousse, 2010, G 9-7293

BONIFACE, 50 idées reçues sur l'état du monde, 2e édition, Paris, A. Colin, 2010, G 9-7292

BONIFACE, MATHOUX, La coupe du monde dans tous ses Etats, Paris, Larousse, 2010, G 9-7411

BONNIN, BEURIER, Les corridors écologiques : vers un troisième temps du droit de la conservation de la nature?, Paris, L'Harmattan, 2008, G 9-7443

BOPP, Les poissons vont-ils mourir de faim (et nous avec) ?, Paris, Le Pommier, 2010, G 9-7351

BOSCHETTI, L' espace culturel transnational, Paris, Nouveau monde, 2010, G 9-7315

BOUCHER, ROYO, LAMY, Les think tanks : cerveaux de la guerre des idées, 2e édition mise à jour et augmentée, Paris, Éd. du Félin-Kiron, 2009, G 9-7323

BOURDIN, L'urbanisme d'après crise, La Tour d'Aigues, Éd. de l'Aube, 2010, G 9-7333

BOUTEFEU, La forêt mise en scène : attentes des publics et scénarios de gestion de la forêt, Paris, L'Harmattan, 2009, G 9-5974

BRILHAC, FAVRO, Planifier le risque industriel, Paris, Victoires, 2009, G 9-7349

BUCKLEY, KALARICKAL,Thirty years of World Bank shelter lending: what have we learned?, Washington, D.C., The World Bank, 2006, G 9-7363

CAMBIER, VAN OORT, SCHVARTZ, DELMAS, Contaminations métalliques des agrosystèmes et écosystèmes péri-industriels, Versailles, Quæ, 2009, G 9-7310

4 CARMIGNANI, Figures du passeur, Perpignan, Presses universitaires de Perpignan, 2002, G 9-7445

CASTARÈDE, Le luxe, 6e édition mise à jour, Paris, Presses universitaires de France, 2010, K 1086 
CHALIAND, Les guerres irrégulières: XXe-XXIe siècles: guérillas et terrorismes, Paris, Gallimard, 2008, G 9-7320

CHALIAND, RAGEAU, Géopolitique des empires : des pharaons à l'imperium américain, Paris, Arthaud, 2010, G 9-7319

CHANDA, LESCOURRET, Au commencement était la mondialisation : la grande saga des aventuriers, missionnaires, soldats et marchands, Paris, CNRS, 2010, G 9-7422

Changements climatiques et sécurité internationale, [S.l.], www.europa.eu, Office des publications, 2008, G 9-7291

CHAUVEAU, Le développement durable : produire pour tous, protéger la planète, 3e édition, Paris, Larousse, 2009, G 9-7379

Le cimetière, Le Bouscat, L'Esprit du Temps, 2009, G 9-7448

COHEN, Richesse du monde, pauvretés des nations, Paris, Flammarion, 1998, G 9-7425

COLLE, D'un protectionnisme l'autre : la fin de la mondialisation? Rapport Anteios 2010, Paris, Presses universitaires de France, 2009, G 9-7337

COLLE, Un monde d'entreprises: rapport Anteios 2009, Paris, Presses universitaires de France, 2008, G 9-7332

COLLET, CARROUÉ, RUIZ, Les mutations de l'économie mondiale de la révolution industrielle à nos jours, 2e édition, Rosny-sous-Bois, Bréal, 2009, G 9-7295

CORBOZ, TIRONI, GREGOTTI, L'espace et le détour : entretiens et essais sur le territoire, la ville, la complexité et les doutes, Lausanne, L'Âge d'homme, 2009, G 9-7442

COURMONT, RIBNIKAR, Les guerres asymétriques: conflits d'hier et d'aujourd'hui, terrorisme et nouvelles menaces, 2e édition revue et augmentée, Paris, IRIS ; Dalloz, 2009, G 9-7325

DARBÉRA, Où vont les taxis ?, Paris, Descartes \& Cie, 2009, G 9-7345

Ecologies à l'oeuvre, Arles ; Versailles, Actes sud ; Ecole nationale supérieure du paysage, 2010, G 9-7455

DAVID, LEBRUN, VASSORT, Footafric : coupe du monde, capitalisme et néocolonialisme, Montreuil, L'Échappée, 2010, G 9-7438

DE BLIJ, MULLER, Global geography, Fourteenth edition, international student version, Hoboken, N.J., J. Wiley, 2010, G 9-7353

DEL CASINO, Social geography: a critical introduction, Chichester, Wiley-Blackwell, 2009, G 9-7355

DAVIS, Dead cities, Paris, Les Prairies ordinaires, 2009, G 9-7451

DEBROISE, Les maladies émergentes : quand les virus voyagent, 2e édition, Paris, Larousse, 2009, G 9-7417

DÉCAMPS, DERCOURT, Evénements climatiques extrêmes : réduire les vulnérabilités des systèmes écologiques et sociaux, Paris, EDP sciences, 2010, G 9-7419

DENHEZ, La nature, combien ça coûte? Pourquoi l'écologie n'est pas l'ennemi de l'économie, Paris, Delachaux et Niestlé, 2007, G 9-7305

DENHEZ, Plus de poisson à la criée : menaces sur les réserves mondiales, Paris, Delachaux et Niestlé, 2008, G 9-7350

DJELLOULI, EMELIANOFF, BENNASR, L'étalement urbain : un processus incontrôlable ?, Rennes, Presses universitaires de Rennes, 2010, G 9-7371 

9-7426 G 9-7342 9-7384 G 9-7303 2010, G 9-7302

DOLLÉ, L'inhabitable capital : crise mondiale et expropriation, Fécamp, Lignes, 2010, G

DUBOIS, Le défi alimentaire : étude géopolitique et géoéconomique des agricultures mondiales, Paris, Presses universitaires de France, 2010, G 9-7385

DUHARCOURT, La conjoncture économique et sociale en 2009, Paris, Direction des Journaux officiels, 2009, G 9-7376

DUTEURTRE, FAYE, L'élevage, richesse des pauvres: stratégies d'éleveurs et organisations sociales face aux risques dans les pays du Sud, Versailles, Quæ, 2009, G 9-7343

FELDMAN, La famine menace-t-elle l'humanité ?, Paris, J.C. Lattès, 2010, G 9-7336

FOURNET-GUÉRIN, SNÉGAROFF, TELLENNE, DAVID, SUISSA, La dissertation de géopolitique: lexique, méthode, sujets, Paris, Presses universitaires de France, 2005,

FRANCHETTI PARDO, COSTA, L'invention de la ville occidentale, Rodez, Éd. du Rouergue, 2008, AQ 9-52 BIS

FRANCONIE, CHASTANET, SIGAUT, Couscous, boulgour et polenta: transformer et consommer les céréales dans le monde, Paris, Karthala, 2010, G 9-7301

FRÉROT, GURRÍA, L'eau : pour une culture de la responsabilité, Paris, Autrement, 2009, G

FUMEY, Manger local, manger global: l'alimentation géographique, Paris, CNRS, 2010,

GAUCHON, HUISSOUD, Les grandes puissances du XXIe siècle: histoire, économie, géopolitique : rapport Anteios 2008, Paris, Presses universitaires de France, 2007, G 9-7318

GAUCHON, HUISSOUD, Les 100 mots de la géopolitique, 2e édition mise à jour, Paris, Presses universitaires de France, 2010, K 1081

GÉRÉ, La nouvelle géopolitique : guerres et paix aujourd'hui, Paris, Larousse, 2005, G 9-7378

GERONIMI, CHOQUET, RANDRIANASOLO, Savoirs et politiques de développement : questions en débat à l'aube du XXIe siècle, Paris, Karthala ; GEMDEV, 2008, G 9-7395

GILLON, GROSJEAN, RAVENEL, Atlas du sport mondial : business et spectacle : l'idéal sportif en jeu, Paris, Autrement, 2010, G 9-7420

GIRARD, DAUM, La géographie n'est plus ce que vous croyez, Talmont-Saint-Hilaire, Codex,

GIROUD, Semences et recherche : des voies du progrès, Paris, Direction des Journaux officiels, 2008, G 9-7374

GOULD, The slow plague: a geography of the AIDS pandemic, Oxford, UK; Cambridge, USA, Blackwell, 1993, G 9-7361

GOURDIN, Géopolitiques : manuel pratique, Paris, Choiseul, 2010, G 9-7421

GRÜNEWALD, LEVRON, Villes en guerre et guerres en ville : pratiques humanitaires en questions, Paris, Karthala, 2004, G 9-7444

GUILLOCHON, La mondialisation : une seule planète, des projets divergents, 3e édition, Paris, Larousse, 2009, G 9-7377

HALL, PAGE,Thegeography of tourism and recreation : environment, place and space, 3è éd., London; New York, Routledge, 2006, G 9-7368 
HAUPTMANN, WATES, Concertation citoyenne en urbanisme : la méthode du Community planning, Gap ; Paris, Y. Michel ; Adels, 2010, G 9-7372

HEROD, WRIGHT,Geographies of power: placinG scale, Malden, Blackwell, 2002, G 9-7356

HERVIEU, Mediterra 2008, Paris, Presses de Sciences Po, 2008, G 9-7341

HILLARD, Bertelsmann: un empire des médias et une fondation au service du mondialisme, Paris, F.-X. de Guibert, 2009, G 9-7299

HILLARD, La marche irrésistible du nouvel ordre mondial : l'échec de la tour de babel n'est pas fatal, Paris, F.-X. de Guibert, 2008, G 9-7298

HOLIFIELD, PORTER, WALKER,Spaces of environmental justice, Malden, Mass., WileyBlackwell, 2010, G 9-7357

HOERNER, Mémoires d'un nouveau touriste, Lyon, Balzac ; CirVath, 2006, G 9-7428

HUBBARD, City, London; New York, Routledge, 2006, G 9-7366

HUGON, L'économie du développement et la pensée francophone, Paris, Ed. des archives contemporaines ; Agence universitaire de la Francophonie, 2008, G 9-7313

HUGON, SALAMA, Les suds dans la crise, Paris, A. Colin, 2009, G 9-7326

Les infortunes de l'espace, Paris La Défense, Secrétariat permanent du Plan Urbanisme Construction Architecture, 2003, G 9-7398

INGALLINA, Le projet urbain, 4e édition mise à jour, Paris, Presses universitaires de France, 2010, K 1087

IRELAND, ZUFFEREY, Le paysage envisagé, Gollion (Suisse), Infolio, 2009, G 9-7454

JAKOB, L'Émergence du paysage, Gollion (Suisse), Infolio, 2004, G 9-7453

JACQUET, PACHAURI, TUBIANA, Villes : changer de trajectoire, Paris, Presses de Sciences po, 2010, G 9-7346

JONES, WILLS,The invention of the park: recreational landscapes from the Garden of Eden to Disney's Magic Kingdom, Cambridge, UK; Malden, MA, Polity, 2005, G 9-7352

KAPLAN, WHEELER, HOLLOWAY, HODLER,Urban geography, 2nd ed., Hoboken, Wiley, 2004, G 9-7354

KOGEJ, Les mutations de l'économie mondiale du début du XXe siècle aux années 1970 en fiches, 2e édition, Rosny, Bréal, 2008, G 9-7296

LACOSTE, La question post-coloniale : une analyse géopolitique, Paris, Fayard, 2010, G 9-7358

LANDAUER, L'architecte, la ville et la sécurité, Paris, Presses universitaires de France, 2009, G 9-7386

LE CLÉZIO, Les indicateurs du développement durable et l'empreinte écologique, Paris, Direction des Journaux officiels, 2009, G 9-7375

LECLERC-OLIVE, Affaires locales: de l'espace social communautaire à l'espace public politique, Paris, Gemdev ; Karthala, 2006, G 9-7300

LEFÈVRE-BALLEYDIER, L'après-pétrole : lorsque les puits seront à sec, 2e édition, Paris, Larousse, 2009, G 9-7380

LÉVY, FAN, JOLY, Economie circulaire: l'urgence écologique: monde en transe, Chine en transit, Paris, Presses de l'Ecole nationale des ponts et chaussées, 2009, G 9-7308

LUNZENFICHTER, BÉBÉAR, Athènes (1896)...Rio (2016) : choix épique des villes olympiques, Biarritz, Atlantica, 2010, G 9-7314 
MAASS, DAZIN, Pétrole brut : enquête mondiale sur une richesse destructrice, Paris, Autrement, 2010, G 9-7383

MAITLAND, NEWMAN,World tourism cities : developing tourism off the beaten track, New York, Routledge, 2008, G 9-7369

MAMONTOFF, HOERNER, Pour une nouvelle recherche en tourisme, Baixas, Balzac, 2009, G 9-7360

MAULNY, PERRUCHE, La guerre en réseau au XXIe siècle : internet sur les champs de bataille, Paris, Ed. du Félin-Kiron, 2006, G 9-7321

MICHAÏLOF, BONNEL, SEVERINO, ORSENNA, Notre maison brûle au Sud: que peut faire l'aide au développement ?, Paris, Fayard ; Commentaire, 2010, G 9-7304

METMATI, L'art de la guerre en réseaux 1991-2009: une nouvelle ère dans l'art de construire une action, Paris, L'Harmattan, 2010, G 9-7423

Mobilités du quotidien, Paris, CERAS, 2008, G 9-7334

La mondialisation en fiches: genèse, acteurs et enjeux, 2e édition, Rosny, Bréal, 2008, G 9-7297

MONGIN ET AL., Le bel avenir de la pauvreté, Paris, Esprit, G 9-7413

MONS, Les enjeux de l'énergie : pétrole, nucléaire, et après ?, 3e édition, Paris, Larousse, 2008, G 9-7382

MUCHNIK, SAINTE MARIE, LECLERC, Le temps des SYAL: techniques, vivres et territoires, Versailles, Quæ, 2010, G 9-7311

L'Occident en débat, Paris, La Documentation française, 2010, G 9-7340

ORTOLLAND, PIRAT, LÉVY, Atlas géopolitique des espaces maritimes: frontières, énergie, transports, piraterie, pêche et environnement: première cartographie exhaustive du plateau continental, 2e édition enrichie et augmentée, Paris, Editions Technip, 2010, G 9-7317

PAQUOT, YOUNÈS, LAUDIER, Philosophie de l'environnement et milieux urbains, Paris, La Découverte, 2010, G 9-7447

PÉLEGRIN-GENEL, Des souris dans un labyrinthe : décrypter les ruses et manipulations de nos espaces quotidiens, Paris, Les Empêcheurs de penser en rond ; La Découverte, 2010, G 9-7450

PITTE, Le génie des lieux, Paris, CNRS, 2010, G 9-7312

POYRAZ, GANDAIS, ASLAN, Les quartiers populaires et la ville : les varoş d'Istanbul et les banlieues parisiennes, Paris, L'Harmattan, 2010, G 9-7427

RAINELLI, LEPETIT, L'agriculture de demain: gagnants et perdants de la mondialisation, Paris, Le Félin-Kiron, 2007, G 9-7322

RAVENSCROFT, BRAMMER, RICHARDS,Arsenic pollution: a global synthesis, Chichester, Malden, Wiley-Blackwell, 2009, G 9-7362

RECLUS, Histoire d'un ruisseau, Gollion ; Paris, Infolio, 2010, G 9-7456

RIGAL-CELLARD, Religions et mondialisation : exils, expansions, résistances, Pessac, Presses universitaires de Bordeaux, 2010, G 9-7347

ROTILLON, Économie des ressources naturelles, Nouvelle édition, Paris, La Découverte, 2010, G 9-7359 
ROULLEAU-BERGER, Migrer au féminin, Paris, Presses universitaires de France, 2010, G 9-7446

SASSEN, GUGLIELMINA, La globalisation : une sociologie, Paris, Gallimard, 2009, G 9-7324

SCARWELL, ROUSSEL, Le changement climatique: quand le climat nous pousse à changer d'ère, Villeneuve-d'Ascq, Presses universitaires du Septentrion, 2010, G 9-7307

SCHNEIER-MADANES, L'eau mondialisée : la gouvernance en question, Paris, La Découverte, 2010, G 9-7344

SCHIRMER, VELASCO-GRACIET, BOISSIÈRE, Atlas mondial des vins : la fin d'un ordre consacré, Paris, Autrement, 2010, G 9-7412

SCHWOB, La pluie artificielle et la dissipation locale des brouillards, Conférence faite le 4 juin 1948 à la Maison de la Chimie, Paris, Office national d'études et de recherches aéronautiques, 1948, G 9-7364

SCIAMA, Le changement climatique : une nouvelle ère sur la Terre, 2e édition, Paris, Larousse, 2008, G 9-7381

SEGAUD, Anthropologie de l'espace : habiter, fonder, distribuer, transformer, 2e édition, Paris, A. Colin, 2010, G 9-7430

SEGUIN, Coup de chaud sur l'agriculture, Paris, Delachaux et Niestlé, 2010, G 9-7306

STOPFORD,Maritime economics, Third edition, London ; New York, Routledge, 2009, G 9-7370

TALANDIER, DAVEZIES, Repenser le développement territorial ? Confrontation des modèles d'analyse et des tendances observées dans les pays développés, La Défense, Plan urbanisme construction architecture, 2009, G 9-7339

TERTRAIS, La guerre, Paris, Presses universitaires de France, 2010, K 1082

Tourismes, Toulouse, Université de Toulouse-Le Mirail, 2009, G 9-7327

THOMAS, Les vulnérables : la démocratie contre les pauvres, Bellecombe-en-Bauges, Éditions du Croquant, 2010, G 9-7416

TRÉMOULINAS, Comprendre la crise, 2e édition, Paris, Bréal, 2010, G 9-7338

TROTIGNON, Comprendre le réchauffement climatique : connaître le phénomène scientifique, mesurer les impacts sur la planète, comprendre les conséquences économiques, Paris, Pearson, 2009, G 9-7452

VEYRET-MEKDJIAN, JALTA, HAGNERELLE, Développements durables : tous les enjeux en 12 leçons, Paris, Autrement, 2010, G 9-7290

Villes en évolution, Paris, La Documentation française, 2010, G 9-5973-<2>

VEGA, DAGORN, Villes mondiales : les nouveaux lieux de pouvoir, Auxerre, Sciences humaines, 2009, G 9-7405

VIVANT, Qu'est-ce que la ville créative ?, Paris, Presses universitaires de France, 2009, G 9-7373

WERNER, WERNER, GOETSCHEL, Les épidémies: un sursis permanent, Neuilly-sur-Seine, Atlande, 1999, G 9-7335

WILLIAMS, METH, WILLIS,Geographies of developing areas : the Global South in a changing world, Oxon ; New York, Routledge, 2009, G 9-7367 
YANAPRASART, Paroles d'acteurs de la mobilité : de la mobilité géographique à la mobilité intellectuelle, Paris, L'Harmattan, 2010, 2009, G 9-7429

ZACHARIE, Refonder les politiques de développement, les relations Nord-Sud dans un monde multipolaire, Lormont, Éditions le Bord de l'eau, 2010, G 9-7348

\section{Géographie physique}

BARBAULT, FOUCAULT, Changements climatiques et biodiversité, Paris, Vuibert; AFAS, 2010, G 8-3263

BARDINTZEFF, Le grand livre des volcans du monde, séismes et tsunamis, S.l., Orphie, 2010, G 8-3279

BLANDIN, Biodiversité : l'avenir du vivant, Paris, Albin Michel, 2010, G 8-3262

BRAHIC, TERREAUX, Evaluation économique de la biodiversité: méthodes et exemples pour les forêts tempérées, Versailles, Quae, 2009, G 8-3267

BULL,Tectonically active landscapes, Chichester UK; Hoboken N.J., Wiley-Blackwell, 2009, G 8-3274

BURT, ALLISON,Sediment cascades: an integrated approach, Chichester, Hoboken N.J., Wiley, 2010, G 8-3273

DELAGE, SCHREFLER, Géomécanique environnementale, sols pollués et déchets, Paris, Hermes science publications ; Lavoisier 2005, G 8-3283

DERRUAU, Les formes du relief terrestre : notions de géomorphologie, 8e édition, Paris, A. Colin, 2010, G 8-3277

DEWAELE, SANLOUP, GILLET, L'intérieur de la Terre et des planètes, Paris, Belin, 2005, G 8-3278

DUCROTOY, La restauration écologique des estuaires, Paris, Tec \& Doc, 2010, G 8-3281

ENCRENAZ, LEQUEUX, Les planètes : les nôtres et les autres : de la Terre aux Exoplanètes, Les Ulis, EDP sciences, 2010, G 8-3271

FIEUX, ANDRIÉ, L'océan planétaire, Paris, Presses de l'ENSTA, 2010, G 8-3265

Gestion durable des sols, Versailles, Quae, 2008, G 8-3268

HINGRAY, PICOUET, MUSY, Hydrologie, Lausanne, Presses polytechniques et universitaires romandes, 2009, G 8-2944-<2>, CD 356

HOODA,Trace elements in soils, Chichester, Wiley, 2010, G 8-3272

JAMBON, THOMAS, Géochimie : géodynamique et cycles : cours et exercices corrigés, Paris, Dunod, 2009, G 8-3276

KANDEL, Le réchauffement climatique, 4e édition mise à jour, Paris, Presses universitaires de France, 2010, K 1088

LÉVÊQUE, Faut-il avoir peur des introductions d'espèces?, Paris, le Pommier, 2008, G 8-3264

PARRIAUX, ARNOULD, Géologie: bases pour l'ingénieur, 2e édition revue et augmentée, Lausanne, Presses polytechniques et universitaires romandes, 2009, G 8-3269, DVD 106

RICE, ROY, RHOADS,River confluences, tributaries and the fluvial network, Chichester, England, Hoboken, NJ, Wiley, 2008, G 8-3275 


\section{Mélanges} 6-348 24-454 24-459 2009, G 24-450

SHAHROUR, GOURVÈS, Reconnaissance des terrains in situ, Paris, Hermes science publ. ; Lavoisier, 2005, G 8-3284

TANGUY, Processus estuariens et littoraux; systèmes d'acquisitions des données, Paris, Hermès, Lavoisier, 2009, G 8-3270

TASSIN, Plantes et animaux venus d'ailleurs : une brève histoire des invasions biologiques, Chevagny-sur-Guye, Orphie, 2010, G 8-3280

DUBET, URDICIAN, Exils, passages et transitions : chemins d'une recherche sur les marges : hommage à Rose Duroux, Clermont-Ferrand, Presses universitaires Blaise Pascal, 2008, G

HINNEWINKEL, "Faire vivre le terroir", AOC, terroirs et territoires du vin : hommage au professeur Philippe Roudié, Pessac, Presses universitaires de Bordeaux, 2010, G 6-349

\section{Monde arabe}

AYEB, La crise de la société rurale en Egypte : la fin du Fellah ?, Paris, Karthala, 2010, G

DROZ-VINCENT, Le Moyen-Orient : histoire et civilisations, Paris, Le Cavalier bleu, 2008, G

FEKI, FICQUELMONT, Géopolitique du Liban: constats et enjeux, Levallois-Perret, Studyrama, 2008, G 24-449

GRESH, REKACEWICZ, Israël, Palestine : vérités sur un conflit, Nouvelle édition actualisée, Paris, Fayard, 2010, G 24-448

MAZZELLA, La mondialisation étudiante: le Maghreb entre Nord et Sud, Paris; Tunis, Karthala ; IRMC, 2009, G 24-447

MARCOU, L'Egypte contemporaine, Paris, Le Cavalier bleu, 2008, G 24-458

MEIER, Le Liban, Paris, Le Cavalier bleu, 2010, G 24-460

MOUTON, DBIYAT, Stratégies d'acquisition de l'eau et société au Moyen-Orient depuis l'Antiquité: études de cas, Beyrouth, Institut français d'archéologie du Proche-Orient,

PIAN, Aux nouvelles frontières de l'Europe : l'aventure incertaine des Sénégalais au Maroc, Paris, La Dispute, 2009, G 24-452

PINTA, La Libye, Paris, Karthala, 2006, G 24-453

SAHSAH, Khouribga, genèse d'une ville minière, 1921-1994, Belfort, Université de Technologie de Belfort-Montbéliard, 2010, G 24-451

\section{Périodiques}

BROLSMA,Effect of climate change on temperate forest ecosystems, Utrecht, Royal Dutch Geographical Society, Faculty of Geosciences, Utrecht Univ., 2010, P 3619/B-396 


\section{Ressources électroniques}

479 GIRAUT, VACCHIANI-MARCUZZO,Territories and urbanisation in South Africa : atlas and geo-historical information system (Dysturb), Bondy, IRD, 2009, CD 351

480 Tourisme en ville : pratiques et attentes des visiteurs européens dans les villes françaises : étude quantitative en ligne auprès des visiteurs allemands, anglais, belges, espagnols, français, italiens, Paris, ODIT France, 2008, CD 352

481 Le tourisme fluvial en France en 2008, Paris, Atout France, 2009, CD 353 